\title{
Nonlinear Amplifier Effects in Communications Systems
}

\author{
Cheng-Po Liang, Student Member, IEEE, Je-hong Jong, Student Member, IEEE, \\ Wayne E. Stark, Fellow, IEEE, and Jack R. East, Member, IEEE
}

\begin{abstract}
This paper introduces a figure-of-merit to investigate tradeoffs between amplifiers and modulation waveforms in complex digital communications systems. Class-AB amplifiers are investigated with a variety of modulation schemes to better understand the relations between amplifier efficiency, amplifier distortion, signal in-band and adjacent channel interference, and power consumption. The goal is to better understand the tradeoffs needed to design low-energy communications systems.
\end{abstract}

Index Terms - Communication systems nonlinearities, interchannel interference, nonlinear distortion, power amplifiers.

\section{INTRODUCTION}

$\mathbf{P}$ OWER amplifiers are an important component in modern communications systems, providing the transmit signal levels needed to overcome the loss between the transmitter and receiver. However, they also introduce problems. The amplifier can consume a major fraction of the power used by the system, both the required transmit power and loss associated with amplifier inefficiency. This power consumption helps define the battery life for mobile communications systems [1]. The power amplifier can also distort the transmitted signal, introducing additional noise within the signal frequency band and generating unwanted frequencies in adjacent channels. This channel interference is tightly controlled by the communications standard being used, and must be reduced if possible.

Amplifiers usually operate as a linear device under smallsignal conditions and become more nonlinear and distorting with increasing drive level. The amplifier efficiency also increases with increasing output power, thus, there is a systemlevel tradeoff between the power efficiency or battery life and the resulting distortion. For most commercial systems, this tradeoff is constrained by interference with adjacent users, thus, amplifier signal levels are reduced or "backed off" from the peak efficiency operating point. The military also has communications requirements, but the parameters can be different. Military communications systems operate in different bands and with different data rates than commercial

Manuscript received December 16, 1998; revised May 1999. This work was supported in part by the Department of Defense Research and Engineering Multidisciplinary University Research Initiative on "Low Power/Low Noise Devices," in part by the Army Research Office under Grant ARO DAAH0496-1-0001, and in part by the Defense Advanced Research Projects Agency and Communications-Electronics Command under Grant SRA-588510732.

The authors are with the Department of Electrical Engineering and Computer Science, The University of Michigan at Ann Arbor, Ann Arbor, MI 48109 USA.

Publisher Item Identifier S 0018-9480(99)06083-4. systems. Mobility and reasonable operating or talk times between battery charges are critical. These, in turn, require low weight and long battery life. Both can be improved with a more power-efficient system.

This paper investigates the tradeoffs between modulation and amplifier nonlinearity and efficiency. The goal is to investigate the amplifier-modulation combination to minimize the energy required to communicate information. The paper is organized as follows. Section II introduces a useful figure-ofmerit to quantify the energy requirements of different amplifier modulation combinations under different drive conditions. Section III describes the system models, including different modulations schemes, and the amplifier model being used. Section IV discusses the simulation procedures and results of the simulations. Finally, the paper is summarized in Section V.

\section{Communications System Performance Measure}

In this section, we first illustrate a conventional performance measure in communication systems. We then present a new performance measure, which takes into account amplifier power efficiency, amplifier nonlinear distortion, modulated signal power efficiency, and signal bandwidth efficiency.

\section{A. Conventional Performance Measure}

The conventional performance measure for a digital communication system is the bit error rate (BER), which is usually a decreasing function of $E_{b} / N_{0} . E_{b}$ is the average received energy per bit and $N_{0} / 2$ is the two-sided power spectral density (PSD) of the noise in an additive white Gaussian noise (AWGN) channel. The relation between $E_{b}$, average received power $\bar{P}_{r}$, and data rate $R_{b}$ is

$$
\frac{\bar{P}_{r} \mathrm{~J} / \mathrm{s}}{R_{b} \text { bits } / \mathrm{s}}=E_{b} \text { joules } / \text { bit. }
$$

One goal is to minimize the required $E_{b} / N_{0}$ for a given BER. This required $E_{b} / N_{0}$ differs for different modulation schemes, as does the PSD function of the modulated signal. Some modulation schemes have a more compact PSD than others, and usually a large data rate $R_{b}$ requires a larger frequency band. An alternative goal is to send as many bits as possible for a given bandwidth $W$ while achieving the prescribed BER. This is usually quantified by the bandwidth efficiency, which is defined as

$$
\frac{R_{b}}{W} \text { bits/s/Hz. }
$$




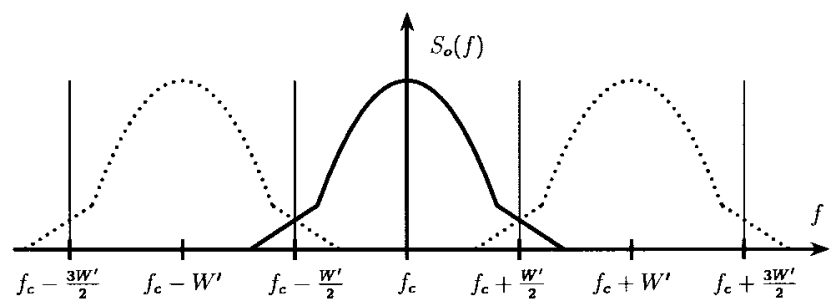

Fig. 1. PSD of the desired signal and adjacent channel signals.

The overall goal is to minimize the required $E_{b} / N_{0}$ and to maximize the $R_{b} / W$ while achieving the required BER.

\section{B. Proposed Performance Measure}

The conventional measure $E_{b} / N_{0}$ only takes into account the received power and offers no information as to how much hardware processing power (e.g., dc power consumption of the amplifier) is required for transmission of an information bit. In this paper, a performance measure, including total energy needed per transmitted bit, and the bandwidth efficiency are defined.

The instantaneous power-added efficiency of the amplifier can be defined as

$$
P_{a e}(t)=\left(P_{\mathrm{rf}}(t)-P_{\mathrm{in}}(t)\right) / P_{\mathrm{dc}}(t)
$$

where $P_{\mathrm{in}}(t)$ and $P_{\mathrm{rf}}(t)$ is the input and output RF signal power of the amplifier, and $P_{\mathrm{dc}}(t)$ is the dc power supplied. The average total power $\bar{P}_{t}$ consumed by the amplifier is

$$
\begin{aligned}
\bar{P}_{t} & =\bar{P}_{\mathrm{dc}}+\bar{P}_{\mathrm{in}} \\
& =\bar{P}_{\mathrm{rf}}\left(1+\overline{\left(1-P_{a e}\right) P_{\mathrm{dc}}} / \bar{P}_{\mathrm{rf}}\right) \\
& =\bar{P}_{\mathrm{rf}}(1+w)
\end{aligned}
$$

where $\bar{X}$ denotes the average value of $X$, and the term $w$ in (4) represent the fractional average power not converted to the RF power. Hence, the total energy needed per transmitted bit $E_{t}$ for a given BER and $N_{0}$ is

$$
\begin{aligned}
E_{t} & =\bar{P}_{t} / R_{b} \\
& =\bar{P}_{r}(1+w) / R_{b} \\
& =E_{b}+E_{w}
\end{aligned}
$$

where we assume no propagation loss (i.e., $\bar{P}_{\text {rf }}=\bar{P}_{r}$ ). $E_{b}$ is the required received energy per bit for a given BER and $N_{0} ; E_{w}$ is the corresponding wasted energy per bit due to inefficiencies in the amplifier. $E_{t}$ takes into account both the radiated RF power and the loss due to the inefficiency of the amplifier. Note that $E_{w}$ decreases with increasing input drive level, whereas the required $E_{b}$ usually increases for a given BER and $N_{0}$. This is because increasing the input drive level improves efficiency up to a peak value, but distorts the signal waveforms.

In this paper, the bandwidth efficiency is defined as $R_{b} / W^{\prime}$ where $W^{\prime}$ is the frequency separation between two adjacent channels, as shown in Fig. 1. Furthermore, we assume that the neighboring two channels use the same amplifier and modulation schemes. This is not only to include the effect of out-of-band interference from the nonlinearities into BER, but also to assess the spectral efficiency of different modulation schemes (especially constant envelope modulations). We will discuss this in more detail in Section IV.

The bandwidth efficiency increases as $W^{\prime}$ decreases for a given $R_{b}$. For a given modulation and data rate, the interference from the adjacent channels increases as $W^{\prime}$ decreases because of the smaller channel separation, resulting in a larger required $E_{b} / N_{0}$ for a given BER.

With energy and bandwidth efficiency as a goal, our performance figure-of-merit $D$ is defined as

$$
D=\left(E_{t} / N_{0}\right) /\left(R_{b} / W^{\prime}\right)
$$

where $\left(E_{t} / N_{0}\right)$ is the required total bit energy-to-noise power density ratio for the target BER (in this paper, $10^{-3}$ ). Our goal is to minimize $D$.

\section{SySTEM MODEL}

The performance measure in (6) depends on the choice of modulation scheme and amplifier. A general bandpass modulated signal $s(t)$ has a form given by

$$
\begin{aligned}
s(t) & =A_{c}(t) \cos \left(2 \pi f_{c} t\right)-A_{s}(t) \sin \left(2 \pi f_{c} t\right) \\
& =A(t) \cos \left(2 \pi f_{c} t+\theta(t)\right)
\end{aligned}
$$

where $A(t)=\left(A_{c}^{2}(t)+A_{s}^{2}(t)\right)^{1 / 2}$ is the envelope, $\theta(t)=$ $\tan ^{-1}\left(A_{s}(t) / A_{c}(t)\right)$ is the phase of the signal, and $f_{c}$ is the carrier frequency. $A_{c}(t)$ and $A_{s}(t)$ are called the in-phase and quadrature components, respectively.

The modulated signal is then amplified, and the output of the amplifier can be expressed as

$$
s_{o}(t)=G(A(t)) \cos \left\{2 \pi f_{c} t+\theta(t)+\Phi(A(t))\right\}
$$

if we assume a bandpass memoryless nonlinear model. In this model, the relationship between the input signal and output signal of the amplifier is described by the two memoryless functions, namely, amplitude $G(A(t))$ (AM/AM) and phase $\Phi(A(t))$ (AM/PM) nonlinearities [2]. In a bandpass model, it is assumed that the harmonics (spectral components centered around $\left.\pm n f_{c}, n=0,2,3,4, \cdots\right)$ generated by nonlinearities are rejected by an ideal zonal filter around the carrier frequency $\left(f_{c}\right)$. In this paper, we assume no AM/PM effects. This is because a solid-state power amplifier (SSPA) has usually negligible $\mathrm{AM} / \mathrm{PM}(\Phi(A(t)) \approx 0)$, and it is found, by computer simulation, that the effect of AM/AM is much more significant than AM/PM [3], [4].

Modulation schemes can be divided into two categories: constant envelope modulation and nonconstant envelope modulation. Constant envelope-modulation schemes have their signal envelope $A(t)$ fixed, whereas nonconstant-modulation schemes $A(t)$ is time varying. Constant envelope-modulation schemes are not affected by a nonlinear amplifier transfer function (AM/AM). However, constant envelope-modulation schemes generally have larger bandwidth than nonconstant envelope modulation for a given data rate. This can cause 


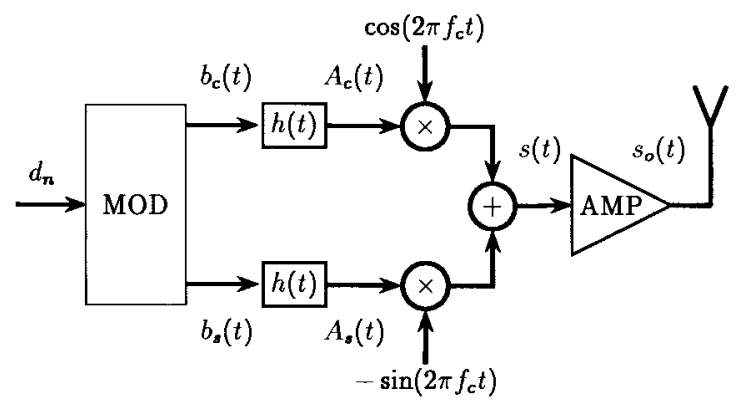

Fig. 2. Transmitter.

TABLE I

PARAMETERS For DiFFERENT MODUlations

\begin{tabular}{l|l|l|l}
\hline Mod & $M$ & $K$ & pulse shaping filter $h(t)$ \\
\hline \hline MSK & 1 & 1 & $\sqrt{2} \cos \left(\frac{\pi t}{T_{2}}\right), \quad-\frac{1}{2} \leq \frac{t}{T_{2}} \leq \frac{1}{2}$ \\
\hline QBL & 1 & 1 & $\left(\sin \left(\frac{\pi t}{T_{t}}\right) / \frac{\pi t}{T_{t}}\right)^{n},-2 \leq \frac{t}{T_{0}} \leq 2$ \\
\hline QPSK & 1 & 0 & square root raised cosine \\
\hline$\pi / 4$ QPSK & 1 & 0 & square root raised cosine \\
\hline OFDM & 64 & 0 & square root raised cosine \\
\hline
\end{tabular}

interference with adjacent channels when the carrier frequency separation between the adjacent channels is small. When nonconstant envelope signals are passed through an amplifier, the amplifier produces both in-band and adjacent channel signals not present in the input waveform, which are a source of interference. An advantage of nonconstant envelope signals is their band-limited nature before nonlinear amplification. At modest drive levels in the linear region of the amplifier, there is little spill over of the output signal into the neighboring channels. These tradeoffs under different operating conditions help determine the energy performance of the amplifier modulation combination. Next, we briefly explain different modulation schemes and the amplifier model used in our investigation.

\section{A. Modulation Schemes}

A block diagram of the transmitter is shown in Fig. 2. The input information data stream with data rate $R_{b}$ (bits/s) to the system is $\left\{d_{n}\right\}$, where $d_{n} \in\{-1,+1\}$. The data is modulated with various modulation schemes to be described below and then passed through a pulse-shaping filter $(h(t))$. The purpose of the pulse-shaping filter is to modify the spectrum of signals, and shape the time-domain properties of digital waveforms. The modulation schemes considered in this paper are constant envelope signals such as minimum shift keying (MSK) [5], modified MSK with quasi-bandlimited pulses (QBL) [6], nonconstant envelope signals such as quaternary phase-shift keying (QPSK) [5], $\pi / 4$ QPSK [7], and orthogonal frequency-division multiplexing (OFDM) [8]. The mathematical representation for each modulation is summarized in the following equations and in Table I.

The in-phase and quadrature component in Fig. 2 are given by

$$
A_{c}(t)=b_{c}(t) * h(t) \quad A_{s}(t)=b_{s}(t) * h(t)
$$

respectively, where

$$
\begin{aligned}
& b_{c}(t)=\sum_{i=-\infty}^{\infty} \sum_{l=0}^{M-1} b_{c, l} \delta\left(t-l T_{s}-i M T_{s}-K T_{s} / 2\right) \\
& b_{s}(t)=\sum_{i=-\infty}^{\infty} \sum_{l=0}^{M-1} b_{s, l} \delta\left(t-l T_{s}-i M T_{s}\right) .
\end{aligned}
$$

The parameters $M, K$, and pulse-shaping filter $h(t)$ are listed in Table I, and $\delta(t)$ is the Dirac delta function. The symbols to be modulated are

$$
\begin{aligned}
& b_{c, l}=\operatorname{Real}\left\{\frac{1}{\sqrt{M}} \sum_{k=0}^{M-1}\left\{d_{c}(k)+j d_{s}(k)\right\} e^{j 2 \pi l k / M)}\right\} \\
& b_{s, l}=\operatorname{Imag}\left\{\frac{1}{\sqrt{M}} \sum_{k=0}^{M-1}\left\{d_{c}(k)+j d_{s}(k)\right\} e^{j 2 \pi l k / M)}\right\}
\end{aligned}
$$

where $d_{c}(k) \in\{-1,1\}$ and $d_{s}(k) \in\{-1,1\}$ are information bits, and $T_{s}=2 R_{b}^{-1}$ is the symbol duration for the all considered modulations, except OFDM; OFDM has a symbol duration of $M T_{s}$.

We discuss only OFDM schemes since this modulation is relatively recent and the details of other schemes can be found in the references. The basic principle behind OFDM is to split a data stream into $M$ streams (serial to parallel process), each of which is transmitted on a separate carrier. In our OFDM system, the number of carrier $M$ is 64 and each carrier is modulated with QPSK. This modulation scheme can be implemented by a discrete Fourier transform (DFT) technique, which eliminates the complexity involved in using a large number of oscillators. This scheme operates block-wise every $M T_{s}$ seconds. That is, $M$ symbols are transmitted every $M T_{s}$ and each symbol constitutes two information bits.

In Table I, the modulation schemes QPSK, $\pi / 4$ QPSK, and OFDM use a square-root raised-cosine filter for $h(t)$ [5, p. 547] with a rolloff factor of 0.35 , which is currently used for North American TDMA Digital Cellular (IS-54/136) [4]. Also note that $\pi / 4$ QPSK is the same as QPSK, except that every other symbol is rotated by $45^{\circ}$. In QBL, the input to the amplifier $s(t)$ is hard-limited, which results in a constant envelope signal. For QBL, the bandwidth of the signal increases with $n$. In this paper, $n=3$ is used according to [6], where the bandwidth is nearly the same as an MSK signal.

\section{B. Amplifier Model}

A class-AB amplifier was used to simulate the power amplifier. FET-based amplifiers were used in these examples. A transistor amplifier biased for class- $\mathrm{AB}$ operation was designed and simulated using Libra to predict the output power (AM/AM) and amplifier efficiency versus input drive level. These are plotted in Fig. 3. As can be seen in the plot, the amplifier is nearly linear at a small input power level, while it becomes nonlinear as the input power level gets large (at an input power below $1 \mathrm{~mW}$, the amplifier can be regarded as a fairly linear amplifier). Again, phase distortion (AM/PM) of the amplifier is not considered in this paper since it is found to be negligible in the simulation results. 

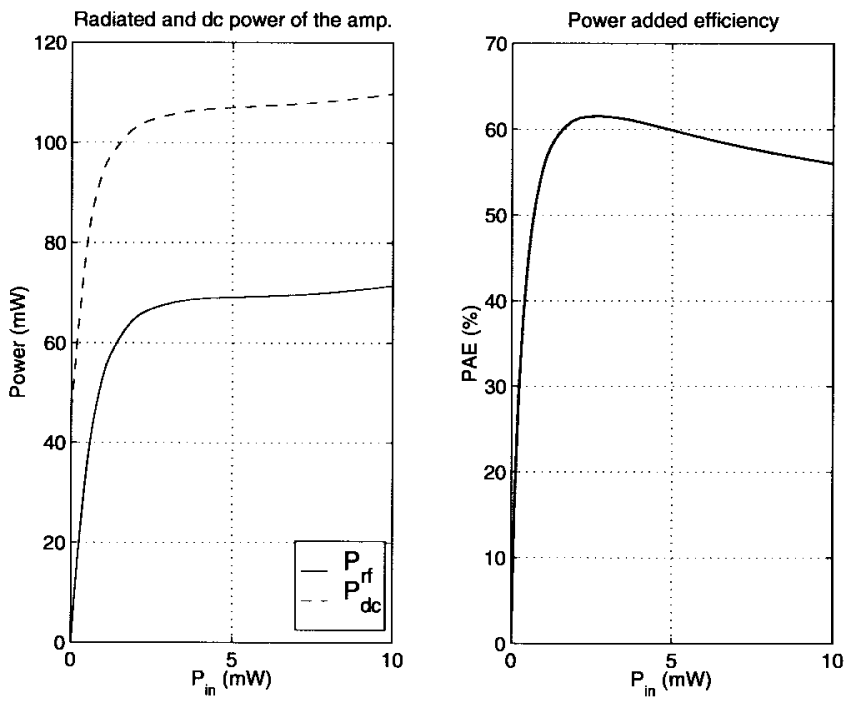

Fig. 3. Amplifier characteristics.

\section{RESULTS}

A Monte Carlo method is used in the computer simulation. Thirty-two samples are taken in each $T_{s}$ s. Waveforms are generated by passing impulse trains through the pulse-shaping filter described in Table I. A table lookup with linear interpolation is used for input-output characteristics of the amplifier. This table is generated by the Libra simulator. After nonlinear amplification, the signal is passed through the AWGN channel where Gaussian noise with two-sided PSD $N_{0} / 2$ is added to it. The received signal is filtered with a matched filter (a filter matched to the signal before the nonlinearities). Due to the nonlinearity, the matched filter is not the optimum receiver. The decision on the data is made after sampling the output of the matched filter, where the demodulated data sequence is compared with the transmitted data sequence to calculate the BER.

In this paper, we quantify the effect of different input drive levels of the amplifier on the in-band distortion, as well as adjacent channel interference (ACI) due to spectral regrowth. Furthermore, with the proposed performance measure, we find an optimum amplifier drive level, in terms of minimizing total energy per bit, for each modulation scheme and bandwidth efficiency. We consider four different channel spacings $\left(W^{\prime} / R_{b}\right.$ $=0.55,0.675,1$, and 1.35). First we examine $W^{\prime} / R_{b}=1.35$ to focus on the in-band signal distortion alone since the effects of ACI are quite small for $W^{\prime} / R_{b}=1.35$ (the additional degradation in $D$ due to ACI is found to be less than $0.1 \mathrm{~dB}$ ). We then investigate a smaller value of $W^{\prime} / R_{b}$ where the ACI becomes more predominant.

In Figs. 4-7, we plot $D$ (decibels) versus the input backoff (IBO) (decibels), which is defined as the ratio of the input saturation power, where the output power begins to saturate, to the average input power

$$
\mathrm{IBO}=10 \log \left(P_{\mathrm{in}}^{\mathrm{sat}} / \bar{P}_{\mathrm{in}}\right) .
$$

Input saturation power in this paper is $2.5 \mathrm{~mW}$. For comparison purposes, an ideal case, the bottom straight line, in each plot

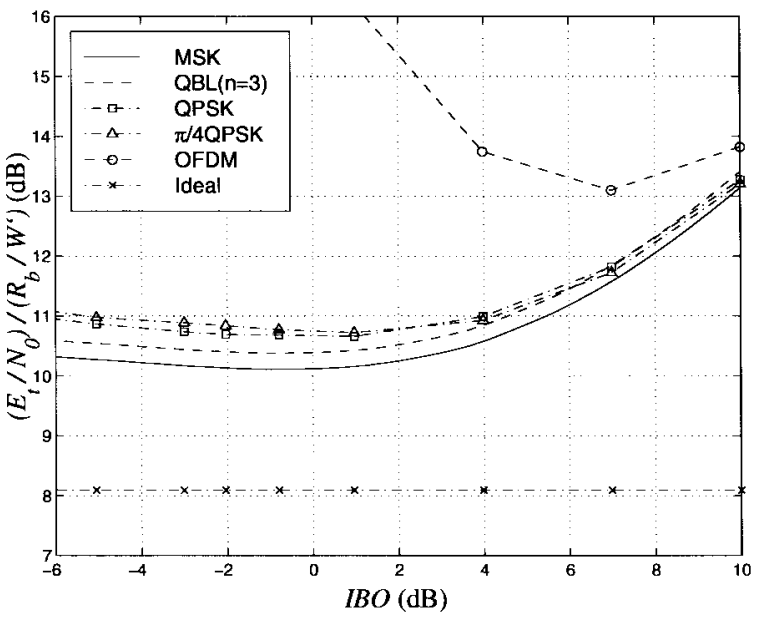

Fig. 4. System performance with $W^{\prime} / R_{b}=1.35$ and $\mathrm{BER}=10^{-3}$,

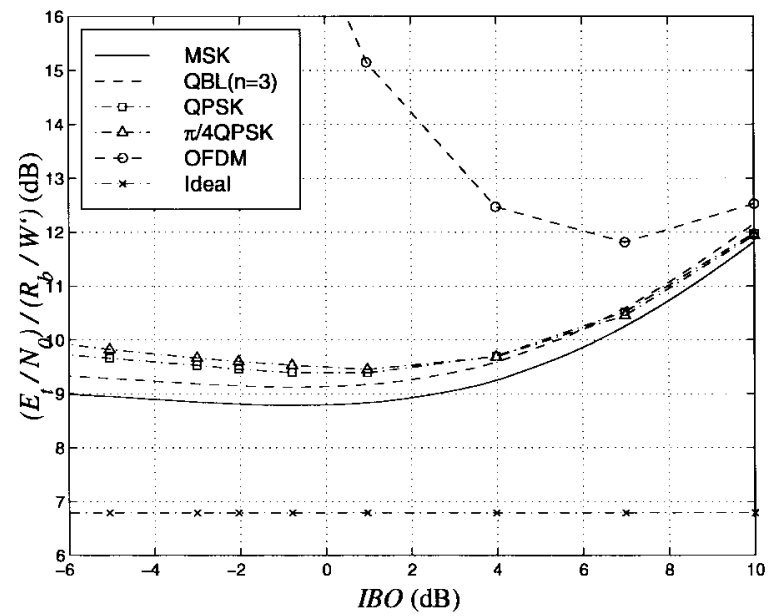

Fig. 5. System performance with $W^{\prime} / R_{b}=1$ and BER $=10^{-3}$.

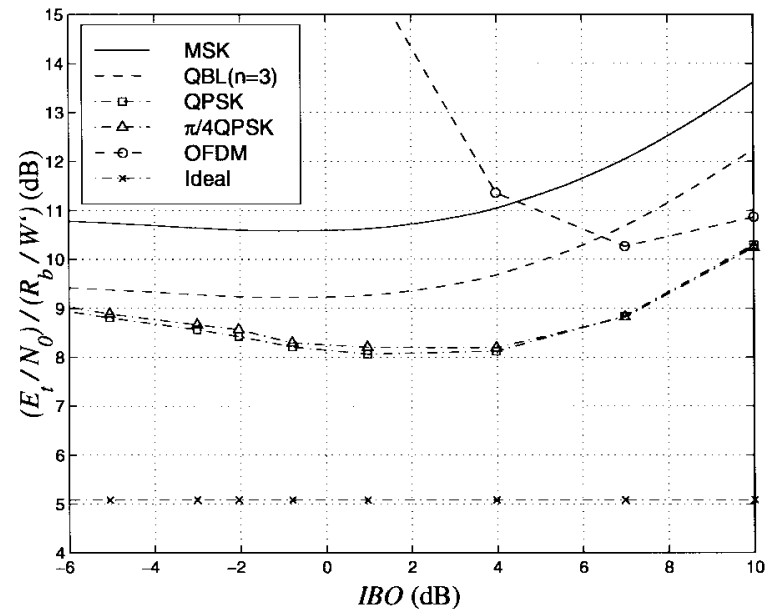

Fig. 6. System performance with $W^{\prime} / R_{b}=0.675$ and $\mathrm{BER}=10^{-3}$.

is obtained, assuming a 100\% efficient linear amplifier and without ACI.

In Fig. 4, for constant envelope signals, the degradation relative to the ideal case is mainly due to inefficiency of the amplifier since there is no signal degradation from the 


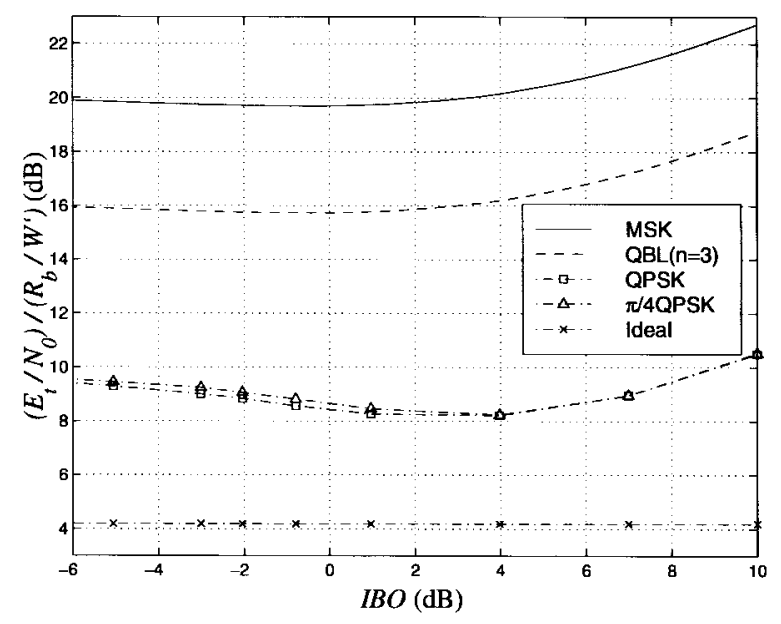

Fig. 7. System performance with $W^{\prime} / R_{b}=0.55$ and $\mathrm{BER}=10^{-3}$.

nonlinear transfer function of the amplifier, and ACI is small. The figure-of-merit $D$ increases, while the IBO is greater than $1 \mathrm{~dB}$. This is because the efficiency of the amplifier is extremely poor at very low input drive level and increases rapidly as indicated in the power-added efficiency (PAE) curve in Fig. 3. After $D$ drops to a minimum, the curves of constant envelope signals begin to rise slowly. This can also be seen from the right-hand-side plot of Fig. 3, which shows that the efficiency drops slowly from $62 \%$ to $56 \%$, while the input drive level goes from $2 \mathrm{~mW}(\mathrm{IBO}=0.97 \mathrm{~dB})$ to $10 \mathrm{~mW}$ $(\mathrm{IBO}=-6 \mathrm{~dB})$.

For nonconstant envelope signals, the effect on $D$ is quite different. While IBO is larger than $4 \mathrm{~dB}$, the amplifier can be regarded as a fairly linear amplifier and the performance loss due to transmitter nonlinearities is found to be less than $0.1 \mathrm{~dB}$ for QPSK and $\pi / 4$ QPSK. However, the amplifier efficiency is relatively low at small-signal driving level. This results in higher overall power and, hence, poorer performance as shown in Fig. 4. Increasing the drive level increases the efficiency and decreases $D$ to a minimum value. While operating in this region, the effect of the improvement in the amplifier efficiency is greater than the effects of the signal degradation due to transmitter nonlinearities. Finally, the efficiency begins to drop for higher drive levels, and the signal distortion becomes significant. The increase of $D$ for a nonconstant envelope signals is a combined effect of amplifier inefficiency and signal distortion from nonlinear amplification.

As can be seen in Figs. 4-6, the performance of OFDM is worst among all modulation schemes when the IBO is less than $4 \mathrm{~dB}$. Note that in Fig. 7, the figure-of-merit $D$ of OFDM is not shown. This is because its value is well above the range of the plot. This is due to the overlap of the adjacent channels, which cause the BER on the subcarriers at the edge of the band to be very large. Even when the frequency separation is large (because OFDM has high envelope variations), the clipping effects of the amplifier on the signal envelope results in more distortion of the signal waveform than other modulation schemes. Even with its poor performance in the presence of amplifier nonlinearities, OFDM is still of considerable interest because of its high immunity to multipath fading channels.
By reducing $W^{\prime} / R_{b}$, adjacent channels are brought closer together, and the ACI effect becomes significant. In Figs. 4-7, we can see that as $W^{\prime} / R_{b}$ decreases, nonconstant envelope signals outperform the constant envelope signals (except OFDM). This is due to the fact that the nonconstant envelope signals are more spectrally efficient than the constant envelope signals in our study. Fast rolloff of the square-root raised-cosine filter has made the nonconstant envelope signal spectrally efficient.

The optimum IBO for QPSK and $\pi / 4$ QPSK are approximately $1 \mathrm{~dB}$ when $W^{\prime} / R_{b}$ is large (Figs. 4 and 5). The optimum drive level gradually moves from 1 to $4 \mathrm{~dB}$ as $W^{\prime} / R_{b}$ decreases. This is due to the fact that as the adjacent channels get closer, the ACI becomes more sensitive to transmitter nonlinearities, and, hence, the more linear the operation, the better performance. When the amplifier is driven hard $(\mathrm{IBO}=-6 \mathrm{~dB})$, the maximum degradation in performance due to nonlinear amplification is less than about $1 \mathrm{~dB}$ (except OFDM). This suggests that the amplifier efficiency is a more dominant effect on $D$ than the linearities of the amplifier, for QPSK and $\pi / 4$ QPSK.

For constant envelope signals, QBL is more compact in spectrum than MSK and, therefore, out performs MSK while $W^{\prime} / R_{b}$ is small. The spectral efficiency of QBL signal lies between nonconstant envelope signals and MSK signals. This is because of the pulse shape used and also the relaxation of the time constraint for its basic pulses. The performance loss due to this relaxation of the constraint is approximately $0.3 \mathrm{~dB}$ with regards to MSK while $W^{\prime} / R_{b}$ is large. Nevertheless, as $W^{\prime} / R_{b}$ decreases, QBL gradually outperforms MSK, which is shown in Figs. 4-7. Generally, both of them perform inferior to QPSK and $\pi / 4$ QPSK at small $W^{\prime} / R_{b}$ because of spectral inefficiency.

\section{CONCLUSIONS}

Bandwidth efficiency and power efficiency are often conflicting criteria. One usually has to be traded off with the other according to the guidelines or the system requirements. In this paper, these tradeoffs are quantified by the new proposed performance measure, and the results are obtained through the computer simulation. This performance measure is also useful for coded systems or systems with other types of receivers.

In terms of commercial products, it is important to accommodate as many users in the system as possible. Therefore, bandwidth efficiency is often the major concern. In this paper, nonconstant envelope signals with pulse-shaping filters (QPSK and $\pi / 4$ QPSK) that have better spectrum efficiency would be a more preferable choice over constant envelope signals (MSK and QBL) for a bandwidth-efficient system. On the other hand, battery life or operating time is often a major concern. In this case, constant envelope signals (MSK) with higher amplifier efficiency that reduce overall processing power would often be a more preferable choice for a power-efficient system. QBL can be regarded as a compromise between bandwidth efficiency system and power efficiency system. This is because of the QBL's modified pulse shape allowing a better spectral usage while retaining the property of being a constant envelope 
signal. OFDM shows its high sensitivities to nonlinear effects, requiring more linear amplification than other modulation schemes considered in this paper.

In this paper, a new performance measure for optimizing communication systems power consumption has been introduced. Furthermore, a simulation tool that evaluates the performance of the nonlinear amplifier interacting with modulated waveforms was developed. This evaluation of the effect of nonlinearities on the modulation can be simulated and optimized prior to including other parts of an overall system.

\section{REFERENCES}

[1] L. E. Larson, "Radio frequency integrated circuit technology for lowpower wireless communications," IEEE Personal Commun. Mag.., vol. 5, pp. 11-19, June 1998.

[2] M. C. Jeruchim, P. B. Balaban, and K. S. Shanmugan, Simulations of Communication Systems. New York: Plenum, 1992.

[3] M. T. Le and L. Thibault, "Performance evaluation of COFDM for digital audio broadcasting part II: Effects of HPA nonlinearities," IEEE Trans. Broadcast., vol. 44, pp. 165-171, June 1998

[4] J. Boccuzzi, "Performance evaluation of nonlinear transmit power amplifiers for North American digital cellular portables," IEEE Trans. Veh. Technol., vol. 44, pp. 220-228, May 1995.

[5] J. G. Proakis, Digital Communications. New York: McGraw-Hill, 1995.

[6] F. Amoroso, "The use of quasi-band-limited pulses in MSK transmission," IEEE Trans. Commun.., vol. COM-27, pp. 1616-1624, Oct. 1979.

[7] K. Pahlavan and A. H. Levesque, Wireless Information Networks. New York: Wiley, 1995.

[8] W. Y. Zou and Y. Wu, "COFDM: An overview," IEEE Trans. Broadcasting, vol. 41, pp. 1-8, Mar. 1995.

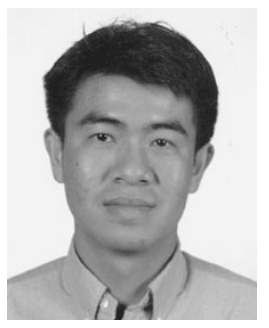

Cheng-Po Liang (S'98) received the B.S. degree in electrical engineering from the National TsingHua University, Hsinchu, Taiwan, R.O.C., in 1994, the M.S. degree in electrical engineering from The University of Michigan at Ann Arbor, in 1998, and is currently working toward the Ph.D. degree in electrical engineering at The University of Michigan at Ann Arbor.

His research interests are in the areas of coding, modulation, nonlinear adaptive filtering and the effect of nonlinear power amplifiers, especially for

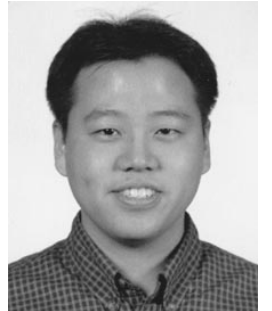

Je-hong Jong (S'97) received the B.S. degree in electronics engineering from Korea University, Seoul, Korea, in 1995, the M.S. degree in electrical engineering from The University of Michigan at Ann Arbor, in 1997, and is currently working toward the Ph.D. degree in electrical engineering at the University of Michigan at Ann Arbor.

During the summer of 1998, he was an Intern with the Hughes Network Systems, MD, where he was involved with the pilot symbol aided trellis coded modulation for the next-generation time-division multiple-access (TDMA) systems. Since 1996, he has been a Research Assistant in the Wireless Communication Laboratory and SolidState Electronics Laboratory, The University of Michigan at Ann Arbor, where his research focuses on spread-spectrum, modulation, error control coding, multicarrier code-division multiple-access (CDMA) systems, nonlinear power amplifiers, and fading channels.

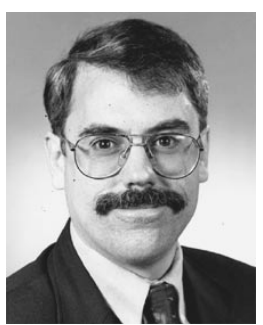

Wayne E. Stark (S'77-M'78-SM'94-F'98) received the B.S. (with highest honors), M.S., and $\mathrm{Ph} . \mathrm{D}$. degrees in electrical engineering from the University of Illinois at Urbana-Champagin, in 1978,1979 , and 1982, respectively.

Since September 1982, he has been a faculty Member in the Department of Electrical Engineering and Computer Science, The University of Michigan at Ann Arbor, where he is currently a Professor. His research interests are in the areas of coding and communication theory, especially for spreadspectrum and wireless communication networks. He is principal investigator of the Army Research Office Multidisciplinary University Research Initiative (MURI) Project on "Low Energy Mobile Communications."

Dr. Stark is a member of Eta Kappa Nu, Phi Kappa Phi, and Tau Beta Pi. He was involved in the planning and organization of the 1986 IEEE International Symposium on Information Theory, Ann Arbor, MI. He was selected by the National Science Foundation as a 1985 Presidential Young Investigator. From 1984 to 1989, he was an associate editor of communication theory for the IEEE TRANSACTIONS ON COMMUNICATIONS in the area of spread-spectrum communications.

Jack R. East (M'74), for biography, see this issue, p. 1432. 